\title{
ASPECTOS PRÁCTICOS DE LA COQUIZACIÓN EN CUNDINAMARCA Y BOYACÁ
}

\author{
(Practical aspects of coking in Cundinamarca y Boyacá)
}

\section{Segundo Manuel Romero Balaguera*}

*Escuela de Ingeniería de Minas, Uptc. Grupo de investigación CEDEMIN.mrmanush9@gmail.com.

\section{(Recibido: 5 de Agosto de 2014 y aceptado 1 de Octubre de 2014)}

Resumen: aprovechando las excelentes calidades de los carbones estudiados de las cuencas carboníferas de Cundinamarca y Boyacá, se abordaron los proyectos de coquización con un cambio en las modalidades de los tipos de hornos de coquización, para obtener un rendimiento excelente y una calidad que les permita ser muy competitivos.

El mercado internacional es muy exigente en cuanto a la calidad del coque y a los índices CRI y CSR, por eso se implementaron laboratorios de carbones y se mejoró el sistema de muestreo y de análisis de los laboratorios por otros más agiles y modernos.

Las mezclas se hicieron con la metodología de prueba y error y métodos empíricos de combinación del índice de hinchamiento, y en el rango de los carbones se tomaron los tres principales tipos: altos, medio y bajo volátiles. Pero con los estudios y el cambio tecnológico y control ambiental se logró mejorar el proceso, lo que permitió que la industria del coque sirviera como polo de desarrollo para el impulso de las industrias en el centro del país.

En el estudio se presenta todo el proceso, iniciando con la explotación de los carbones coquizables, los cuidados que deben tenerse en cuenta para mantener la buena calidad, el transporte y medidas ambientales, el proceso de trituración y molienda para preparar la mezcla óptima y los ensayos de laboratorio necesarios para mantener el control de calidad, los tipos de hornos existentes en Boyacá, Colombia, los rendimientos y adelantos en esas tecnologías, y finalmente, el proceso de apagado del sistema. Todo lo anterior se abordó con el fin de darle valor agregado al producto con el cumplimiento de los estándares del mercado internacional

Palabras clave: coquización, explotacion, trituración molienda, hornos de carbón, calidad.
Abstract: taking advantage of the excellent qualities of the studied coals from the coalfields in Cundinamarca and Boyacá, coking projects were addressed with a change in the terms of the types of coking ovens, for excellent performance and quality that allows them to be very competitive.

The international market is very demanding in terms of the quality of coke and CRI and CSR indexes, that is why coals laboratories were implemented and improved the system of sampling and analysis of laboratories for more agile and modern.

Mixtures were made with the test methodology and empirical methods combining swelling index and rank coals were the three main types: high, medium and low volatile. But with studies and technological change and environmental control, process is managed, allowing the coke industry to serve as a development center for the promotion of industries in the central area of this country.

In the study the whole process is presented beginning with the exploitation of coking coals, the care that must be taken into account to maintain good quality, transport and environmental measures, the process of crushing and grinding to prepare the optimal mix and laboratory tests necessary to maintain quality control, the types of ovens in Boyacá Colombia, yields and advances in these technologies, and finally, the system shutdown process. All of the above was addressed in order to add value to the product with the fulfillment of the international market standards.

\section{Keywords: coking, exploitation, crushing, grinding, coal} furnaces, quality.

\section{INTRODUCCIÓN}

El altiplano cundiboyacense y los santanderes son poseedores de muy buenas reservas de carbón metalúrgico en Colombia. Durante las dos últimas décadas se ha realizado un interesante número de investigaciones tendientes a la caracterización de estos carbones y a plantear las diferentes mezclas, para obtener, mediante el proceso de coquización, el producto óptimo en el mercado internacional. Por tal razón, la UPTC y la empresa privada, en una alianza estratégica, se propusieron adelantar todo lo necesario para que se implementara la mejor tecnología para la producción del coque exigido en el mercado.
El mercado internacional del coque es el futuro de las explotaciones de carbones latinoamericanos, siempre que se realice de manera técnica, económica, social y ambientalmente sostenible.

\section{TECNOLOGÍA GENERAL DE LA COQUIZACIÓN}

El fenómeno de la coquización es aquel en que un carbón de un rango definido ablanda por calentamiento, se hincha y se resolidifica durante la desgasificación continua. 
La coquización consiste en someter a una mezcla de carbones de características adecuadas a destilación o calentamiento en ausencia de aire en horno para tal fin.

La desvolatilización es un proceso continuo, pero se puede hacer una distinción entre la primera etapa de la carbonización, en la cual principalmente se produce alquitrán, y la segunda, en la cual se desprende gas quedando como producto sólido el coque.

Balance: en la carbonización se obtiene:

- Un producto sólido: coque

- Alquitrán: producto líquido compuesto de hidrocarburos y licor acuoso.

- Gases: aprovechamiento de subproductos.

El balance energético provee cerca del $70 \%$ en sólidos, $22 \%$ en alquitrán y el resto en gases.

\section{PIRÓLISIS DEL CARBÓN}

La pirólisis es el proceso de calentar el carbón en ausencia de aire, obteniendo de esta forma una ruptura de la molécula, que se traduce en productos químicos que se desprenden y un residuo (el coque) que se va acumulando y transformando en la misma medida.

\subsection{Características del carbón para coquización:}

La designación de un carbón como coquizable o no, depende de su comportamiento cuando se calienta en ausencia de aire. Si se ablanda y eventualmente se solidifica en una masa más o menos compacta, se clasifica como coquizable.

\section{La producción de un buen coque depende:}

a.- Del carácter de la sustancia del carbón que conduce a la formación de la estructura celular con las cualidades apropiadas.

b. De la calidad del carbón que define la proporción de azufre y cenizas en ambos.

Los carbones para coquización se clasifican mediante ensayos para medir propiedades coquizantes: FSI, plastometría, dilatometría. Propiedades aglutinantes: índice Roga, ensayo Gray-King, petrografía.

Diferentes carbones muestran marcadas diferencias en sus características plásticas y dilatoras, que dependen del rango del carbón, la composición petrográfica, la velocidad de calentamiento, la naturaleza del medio de calentamiento.

\section{Valor de uso de un carbón para coquización:}

Las características de un carbón para garantizar coquización son:

$\%$ Vt entre 30 y $75 \%$, preferiblemente $>60 \%$

Cen Vt 84 y $91 \%$

PRV 0.8 y 1.8

FSI $>3 \frac{1}{2}$

Plasticidad $>10$ DDPM

Dilatación libre entre 50 y $140 \%$
Además, para obtener coque de buena calidad, se requiere:

$\% \mathrm{MV} 18$ y $32 \%$

$\mathrm{S}<1.0 \%$

Cenizas $>10 \%$

$\mathrm{P}>0.03 \%$

\section{COMPORTAMIENTO DEL CARBÓN AL CALENTARSE EN AUSENCIA DE AIRE}

Cuando el carbón se calienta con suficiente aire, se presenta la combustión. Pero si el carbón coquizable se calienta en ausencia de aire, tiene lugar un fenómeno característico:

- El reblandecimiento temporal y la resolidificación de la materia (siempre a temperaturas crecientes).

En ambos periodos hay desprendimiento de gases: desgasificación primaria durante el reblandecimiento y secundaria después de la resolidificación.

\subsection{Sucesión de fenómenos de transformación de carbón en coque}

A una temperatura que comienza poco después de $100^{\circ} \mathrm{C}$ se desprende $\mathrm{H} 2 \mathrm{O}$ del carbón, en el siguiente orden.

$\begin{array}{ll}- & \text { H2O inhibición } \\ \text { - } & \text { H2O retenida en poros } \\ \text { - } & \text { H2O composición, químicamente } \\ \text { - } & \text { H2O gases ocluidos }\end{array}$

Aunque no se puede hablar de procesos discontinuos, se advierte que la velocidad de desprendimiento de las materias gaseosas, aumenta violentamente entre $350{ }^{\circ} \mathrm{C}$ y $550{ }^{\circ} \mathrm{C}$, en primer lugar, y después de casi anularse, vuelve a aumentar cerca de los $700^{\circ} \mathrm{C}$.

El primer máximo es lo que constituye la desgasificación primaria, y los gases desprendidos son principalmente carbono, hidrógeno y oxígeno. El segundo máximo es la desgasificación secundaria desprendiendo solo hidrógeno.

Hacia los $400{ }^{\circ} \mathrm{C}$, el carbón se reblandece aumentando su plasticidad poco a poco; a $400{ }^{\circ} \mathrm{C}$, la masa reblandecida comienza a hincharse, sin dejar de desprender gases, este fenómeno da una estructura esponjosa.

Al pasar $600{ }^{\circ} \mathrm{C}$ comienza la coagulación, posteriormente sigue el endurecimiento, para finalizar después de aglomerarse fuertemente la materia carbonosa ya solo constituida por carbones, con una ligera contracción en volumen "el coque se ha formado".

Para carbones no coquizables puede suceder: si no existe desprendimiento suficiente de gases (antracita), no hay esponjamiento alguno. Si no hay hinchamiento o fusión, no hay coque; la falta de aglomeración produce coque incoherente y pulvurento. 


\section{PRINCIPIOS GENERALES PARA OBTENER EN LA PREPARACIÓN MECÁNICA UN BUEN CARBÓN DE COQUE}

\subsection{Granulometría}

El carbón se muele generalmente a tamaños menores de $6 \mathrm{~mm}$; el molido puede ser metódico o antimetódico.

Molido metódico: no hay tamaños superiores a $6 \mathrm{~mm}$, más costoso, disminuye la capacidad de instalación, produce mayor cantidad de finos.

Molido antimetódico: se aceptan porcentajes de tamaños superiores a $6 \mathrm{~mm}$, estos producen texturas del coque inconvenientes.

Modernamente se ha trabajado a $0,3 \mathrm{~mm}$. En estas condiciones la estructura del coque y su dureza son mejores. En estos límites pueden existir excesos de finos, siempre inconveniente en el caso de mezclas pobres en bitúmenes.

\subsection{Homogeneidad en la carga}

Supuesta una separación de componentes petrográficos (fusibles e infusibles), debe cuidarse la homogeneidad de tamaños de cada uno, y en el caso de infusibles, debe conseguirse la mayor finura.

Una vez preparada la carga, es de gran importancia que la caída en el horno no destruya su homogeneidad o que el movimiento en los silos no de lugar a separaciones de tamaño que resulten en cargas desiguales en retortas.

\subsection{Densidad de carga}

Es el peso de carbón por $\mathrm{m} 3$, es una variable que ejerce considerable influencia en la coquización y que puede variarse según las condiciones industriales; influye en la calidad aunque despreciable en la cantidad de coque producido. Interviene en las presiones sobre las paredes de la retorta, es del orden de 0.80000 ton $/ \mathrm{m} 3$ en el campo industrial. A mayor densidad de carga, se aumenta la producción, se mejora la estabilidad y se presentan tamaños más grandes.

Los factores que influyen en la densidad de carga son: la humedad y la granulometría.

\section{NATURALEZA Y PORCENTAJE DE OTROS CONSTITUYENTES EN LA MEZCLA DECARBONES}

Algunos carbones presentan magníficas propiedades coquizantes en su estado natural, otros pueden convertirse en buenos carbones para coque por el mezclado científico de calidades o por la inclusión en la mezcla de carbones pretratados o por adición de aglutinantes.

Un mezclado tiene los siguientes objetivos:

- Mejoramiento de la calidad de coque.

- Disminución de la presión de hinchamiento.

- Mejoramiento de la reactividad del coque.

- Reducción del tiempo de carbonización

\subsection{Mezcla de aceites minerales con el carbón de coque.}

Se comprobó que la adición del $5 \%$ de aceites minerales al carbón, mejora la resistencia del coque y aumenta los rendimientos de subproductos, benzol, etileno y otros.

Los aceites empleados pueden ser: petróleo, fuel- oil, asfaltos, alquitranes y breas de distintas procedencias.

La adición de aceites mejora el hinchamiento y la aglomeración. El aumento de la plasticidad se origina por la disolución del carbón en el elemento oleoso.

Aumenta el espesor de la zona plástica hasta cierto límite. La resistencia mecánica del coque resultante no es siempre proporcional al espesor de la zona plástica.

La adición de un $5 \%$ de aceites minerales, duplica aproximadamente la resistencia a la abrasividad y aumenta su solidez.

Mejora la densidad de carga haciéndola más regular con independencia de la humedad del carbón.

\subsection{Briqueteado del carbón para uso directo en el alto horno}

La utilización de briquetas, coque moldeado o preformado, se da con el objeto de ampliar la gama de los carbones coquizables y consta de tres etapas:

- Preparación del carbón y semicoque

- Prensado y molido

- Endurecimiento y coquización por calor

A fin de mejorar las condiciones de carga y ampliar el campo de carbones utilizables, sin llegar al apisonado de toda la carga, en algunos casos se procede a prensar o briquetear una parte del carbón que se carga en los hornos realizando luego la coquización de un modo normal; esta técnica se aplica principalmente para aprovechar una mayor gama de carbones, incluso los no coquizantes, en la obtención de coque siderúrgico.

\subsection{Precalentamiento del carbón}

Consiste en efectuar un calentamiento del carbón a $200{ }^{\circ} \mathrm{C}$. El carbón fuera de los hornos y este carbón precalentado se cargan directamente a los hornos convencionales. Este sistema supone: acortamiento en el tiempo de coquización por gran homogeneidad de carga, al mismo tiempo mejora la densidad de carga, lo que significa:

- Aumento en la producción hasta 50 a $60 \%$.

- Mejora de la calidad del coque o posibilidad de emplear mezclas de inferior rango para obtener la misma dureza.

- Supresión total en la contaminación de la carga.

- Uniformidad del coque y perfecta coquización.

- Disminución del consumo energético del 10 al $12 \%$.

- Menor inversión para la misma producción. 


\section{HORNOS DE COQUIZACIÓN}

\subsection{Hornos Pampa}

Descripción: es un recinto de ladrillo dentro del cual se coloca una capa de carbón; después se cubre con polvo de coque húmedo o cenizas. En un lado del recinto hay huecos en la pared que sirven para el encendido y permiten la llegada del aire para la combustión; se presentan chimeneas naturales que se forman en la masa del carbón y que permiten el desprendimiento de humos.

El tiempo de coquización es del orden de diez días sin adicionar los dos días de enfriamiento. En estos hornos la transmisión de calor se hace desde el túnel donde se inicia la combustión hacia lo alto y lo bajo.

En la actualidad no se está utilizando este tipo de hornos por restricciones ambientales, fundamentalmente.

\subsection{Hornos colmena}

Descripción: de forma circular. Con techo en forma de colmena que sirve para irradiar el calor. Tienen un diámetro de 3,60 m promedio con capacidad hasta de 6 ton de carbón. Una puerta lateral sirve para deshornar el coque. Un portillón en la parte superior de la puerta sirve para nivelar el carbón y graduar la entrada de aire. En su parte superior hay una boca por donde se carga el carbón y permite el escape de los gases. Al final del periodo de coquización es apagado con el coque aún dentro del horno y su deshorne es manual.

Están construidos con ladrillo común o arcilla cruda. El piso generalmente es plano y con pendiente hacia la puerta de salida.

Operación y control: se requiere mucho cuidado durante los cambios de condiciones atmosféricas, para mantener la temperatura óptima en el proceso de coquización. Las condiciones individuales de cada horno, el control de la admisión de aire, la cantidad de carbón cargado, la correcta nivelación y la observancia de un buen programa de operación, son fundamentales para manejar la calidad del coque.

El rendimiento del coque por tonelada de carbón en estos hornos varía entre 55 y $65 \%$, dependiendo de los factores anteriormente citados.

\subsection{Hornos de solera}

Descripción: son una modificación de los hornos de colmena, en busca de mejorar la calidad del producto y las condiciones de operación. Son de forma rectangular, de aproximadamente 2.75 por $10.5 \mathrm{~m}$ de largo. Tienen puerta de lado y lado por donde el coque es evacuado por medio de una empujadora o barra deshornadora. Las puertas son quitadas corriéndolas a los lados, presentan huecos de captación de los gases en el techo que los llevan a un sistema de canales en el piso, donde combustionan con el aire suministrado lateralmente por conductos, y descargan los humos a un canal lateral.
Su rata de coquización es de una pulgada por hora y se pueden alcanzar temperaturas hasta $1500^{\circ} \mathrm{C}$.

Control de operación: con programas de cargue y deshorne, con un riguroso y permanente control de temperaturas, en los diferentes sitios donde se pueden alcanzar temperaturas tan altas que hasta se puede fundir el refractario.

La combustión del gas en los canales de recirculación que calientan el piso y la regulación del aire, es un control que debe hacerse con los análisis de los humos determinando el porcentaje de $\mathrm{CO} 2$, de $\mathrm{O} 2 \mathrm{y}$ del $\mathrm{CO}$, o bien por las temperaturas de las llamas determinadas por pirómetros ópticos. Por la falta de control pueden ocurrir explosiones dentro de los diferentes ductos. El tiempo de coquización es de 36 horas y se obtiene un rendimiento entre el 65 y $70 \%$.

\subsection{Hornos de subproductos. Batería}

Descripción: son cámaras largas y estrechas de material refractario, en las cuales el carbón se calienta hasta la temperatura de coquización, por la combustión de gases que pasan por canales abiertos en el refractario de las paredes que limitan las cámaras, entre cada dos retortas sucesivas.

Los hornos modernos para coque metalúrgico tienen una capacidad de 16 a 20 toneladas de carbón por retorta. Las dimensiones de estas varían entre 11 y 13 m de longitud, 35 a 60 $\mathrm{cm}$ de anchura y 3.5 a $6 \mathrm{~m}$ de altura. La velocidad media de coquización es de 17 horas, se pueden tratar alrededor de 24 ton de carbón por día y cámara. En la parte superior de las retortas se abren de tres a cinco orificios de carga de diámetro poco inferior a la anchura de la retorta, protegidos por placas de cierre. A través de ellas se hace la carga del carbón utilizando una máquina de carga que lleva tantas tolvas como orificios tiene una retorta, cuyas bocas se adaptan entre sí. La máquina que se desplaza sobre carriles tiene un sistema de dosificación por peso.

\section{CONCLUSIONES}

Se considera que el proceso de la coquización tiene en los actuales momentos un interesante repunte en el desarrollo tecnológico en el altiplano cundiboyacense, pero se ha suprimido totalmente la fabricación de coque en hornos de pampa por considerarse de alto impacto ambiental.

Los aspectos prácticos de la coquización se reflejan en la manipulación ágil y simple que hacen las empresas existentes en la zona de Guachetá y Samacá, donde se produce la mayor cantidad de coque de alta calidad para ser exportado y consumido en el mercado nacional por importantes empresa como Coque Coal, Carbones Andinos, Milpa y Acerías Paz del Río.

Actualmente se encuentran en operación en la fabricación de coque tres principales tipos de hornos: de colmena, de solera y de batería. Los hornos de pampa ya no deben seguir funcionando más, por ser muy contaminantes para el ambiente. 


\section{REFERENCIAS}

Alzate, C. \& Lopera, N. (2000). Activación de carbones colombianos previamente oxidados, TDG. Medellín: Universidad Nacional de Colombia.

Barraza, J. M. (1995). Liquefaction of beneficiated coal Fractions. $\mathrm{PhD}$ Thesis. England: University of Nottingham.

Enciso, H. et al. (1997). Manual de métodos de predicción de la calidad del coque. Bogotá: Ingeominas.

González, J.A. (1996). Selección de mezclas de carbones-breas para grafitización.
Pis, J. J. et al. (1998). Preparation of active carbons from Coal Part III, Activation of Char, fuel Processing Technology. Volumen 57.

Sirgado, M. (1991). Curso sobre optimización de mezclas coquizables de carbón para eco carbón.

Zimmerman, R. E. (1979). Evaluating and testing the coking properties of coal. San Francisco: ED MFP Inc. 